\title{
MULTIPLE POSITIVE SOLUTIONS FOR PERIODIC BOUNDARY VALUE PROBLEM VIA VARIATIONAL METHODS
}

\author{
YU TIAN AND WEIGAO GE
}

\begin{abstract}
In this paper, we investigate the positive solutions of periodic boundary value problem. By using critical point theory the existence of multiple positive solutions is obtained.
\end{abstract}

\section{Introduction}

In this paper, we consider the existence of multiple positive solutions for the following boundary value problem

$$
\left\{\begin{array}{l}
x^{\prime \prime}(t)-M x(t)+f(t, x(t))=0, \quad t \in[0,2 \pi] \\
x(0)-x(2 \pi)=x^{\prime}(0)-x^{\prime}(2 \pi)=0
\end{array}\right.
$$

where $M>0, f:[0,2 \pi] \times[0,+\infty] \rightarrow[0,+\infty)$ is continuous, $f(t, 0) \not \equiv 0$ for $t \in[0,2 \pi]$ and $F(t, x)=$ $\int_{0}^{x} f(t, s) d s$.

Recently, periodic boundary value problems for differential equations and systems have been studied intensively, see [3], [9], [10], [11], [13] and the references therein. The results have been obtained mainly by the upper and lower solutions method, fixed point theorem in cones.

Jiang [9] applied the Krasnosel'skii's fixed point theorem to the problem (1.1), and get the existence of one positive solution by imposing the following conditions on $f$ :

$$
\lim _{u \rightarrow 0} \max _{t \in[0,2 \pi]} \frac{f(t, u)}{u}=0 \text { and } \lim _{u \rightarrow \infty} \min _{t \in[0,2 \pi]} \frac{f(t, u)}{u}=+\infty \text { (superlinear) }
$$

or

$$
\lim _{u \rightarrow 0} \min _{t \in[0,2 \pi]} \frac{f(t, u)}{u}=+\infty \text { and } \lim _{u \rightarrow \infty} \max _{t \in[0,2 \pi]} \frac{f(t, u)}{u}=0 \text { (sublinear) }
$$

Since the appearance in 1978 of the work of Rabinowitz [14], many authors have considered to apply variational methods to periodic boundary value problem, see [2], [4], [6], [7], [16], [15]. Tang and Wu [16] studied the problem

$$
\left\{\begin{array}{l}
\ddot{u}+\nabla H(t, u)=0, \quad t \in[0, T] \\
u(0)=u(T), \quad \dot{u}(0)=\dot{u}(T) .
\end{array}\right.
$$

Received September 21, 2006.

2000 Mathematics Subject Classification. 34B15, 58E30.

Key words and phrases. Periodic boundary value problem, critical point theory, variational methods.

Supported by grant 10371006 from National Natural Sciences Foundation of P.R. China and grant 20050007011 from Foundation for PhD Specialities of Educational Department of P.R. China. 
where $\int_{0}^{T} b(t) d t=0$, by applying Mountain Pass Theorem, the existence of one solution was obtained. Chen and Long [4] obtained the existence of one solution for the problem (1.4) where $H$ is neither convex nor homogeneous.

As far as we known, there are few papers to consider the existence of multiple positive solutions for periodic boundary value problem by using variational methods. It is well known that positive solutions are very important in applications. In [1], the existence of multiple positive solutions for the following discrete boundary value problem were obtained

$$
\left\{\begin{array}{l}
\Delta^{2} y(k-1)+f(k, y(k))=0, \quad k \in[1, T], \\
y(0)=0=y(T+1),
\end{array}\right.
$$

where $[1, T]$ is the discrete interval $\{1,2, \ldots, T\}, \Delta y(k)=y(k+1)-y(k), f \in C([1, T] \times[0, \infty), R)$ satisfies $f(k, 0) \geq 0, \forall k$. They applied critical point theory and supposed the following conditions hold

(a) $\min _{k \in[1, T]} \liminf _{u \rightarrow \infty} \frac{f(k, u)}{u}>\lambda_{1}$,

$\lambda_{1}$ is the smallest eigenvalue of $\Delta^{2} y(k-1)+\lambda y(k)=0, y \in H$;

(b) there is a constant $M>0$, independent of $\lambda$, such that $\|y\| \neq M$ for every solution $y \geq 0$ to $\Delta^{2} y(k-1)+\lambda f(k, y(k))=0, y \in H$ for each $\lambda \in(0,1]$.

Condition (b) is not easy to be verified in applications.

Our aim of this paper is to apply critical point theory and operator theory to problem (1.1) and obtain new assumptions on nonlinearity of $f$, which are different from superlinear (1.2) or sublinear (1.3) that were obtained by using topological degree theory. This is the first paper to combining variational methods with operator theory to deal with periodic boundary value problem. Moreover, the conditions on $f$ is easy to be verified.

\section{Related lemmas}

Lemma 2.1. Let $x^{+}=\max \{ \pm x, 0\}$, then the following three properties hold:

(i) $x=x^{+}-x^{-}$;

(ii) $\left\|x^{+}\right\| \leq\|x\|$;

(iii) if $\left(x_{n}\right)$ uniformly converges to $x$ in $C([0,2 \pi])$, then $\left(x_{n}^{+}\right)$uniformly converges to $x^{+}$in $C([0,2 \pi])$;

(iv) $x^{+}(t) x^{-}(t)=0,\left(x^{+}\right)^{\prime}(t)\left(x^{-}\right)^{\prime}(t)=0$ for $t \in[0,2 \pi]$.

Lemma 2.2. If $x \in C^{1}([0,2 \pi])$ is a solution of problem

$$
\left\{\begin{array}{l}
x^{\prime \prime}(t)-M x(t)+f\left(t, x^{+}(t)\right)=0, \quad t \in[0,2 \pi], \\
x(0)=x(2 \pi), \quad x^{\prime}(0)=x^{\prime}(2 \pi),
\end{array}\right.
$$

then $x(t) \geq 0, x(t) \not \equiv 0, t \in[0,2 \pi]$ and hence it is a solution of boundary value problem (1.1).

Proof. If $x \in C^{1}([0,2 \pi])$ is a solution of (2.1), then by Lemma 2.1 we have

$$
0=\int_{0}^{2 \pi}\left[x^{\prime \prime}(t)-M x(t)+f\left(t, x^{+}(t)\right)\right] \times x^{-}(t) d t
$$




$$
\begin{aligned}
& =-\int_{0}^{2 \pi}\left[x^{\prime}(t)\left(x^{-}\right)^{\prime}(t)+M x(t) x^{-}(t)\right] d t+\int_{0}^{2 \pi} f\left(t, x^{+}(t)\right) x^{-}(t) d t \\
& \geq \int_{0}^{2 \pi}\left\{\left[\left(x^{-}\right)^{\prime}(t)\right]^{2}+M\left[x^{-}(t)\right]^{2}\right\} d t,
\end{aligned}
$$

so $x^{-}(t)=0$ for $t \in[0,2 \pi]$, that is $x(t) \geq 0$. If $x(t) \equiv 0$, the fact $f(t, 0) \not \equiv 0$ gives a contradiction.

Remark 2.1. By Lemma 2.2, in order to find the positive solutions of boundary value problem (1.1) if suffices to get solutions of (2.1).

Throughout this paper, $X$ denotes the Sobolev space

$$
H_{2 \pi}^{1}([0,2 \pi])=\left\{x \in C([0,2 \pi]) \mid x^{\prime} \in L^{2}([0,2 \pi]), x(0)=x(2 \pi)\right\}
$$

equipped with the norm

$$
\|x\|=\left(\int_{0}^{2 \pi}\left|x^{\prime}(t)\right|^{2}+M|x(t)|^{2} d t\right)^{\frac{1}{2}}
$$

and inner product

$$
\langle x, y\rangle=\int_{0}^{2 \pi}\left[x^{\prime}(t) y^{\prime}(t)+M x(t) y(t)\right] d t .
$$

Clearly $X$ is a reflexive Banach space. Define the functional $\Phi$ on $X$ by

$$
\Phi(x)=\frac{1}{2} \int_{0}^{2 \pi}\left[\left|x^{\prime}(t)\right|^{2}+M|x(t)|^{2}\right] d t-\int_{0}^{2 \pi}\left[F\left(t, x^{+}(t)\right)-f(t, 0) x^{-}(t)\right] d t .
$$

Then the functional $\Phi$ is a continuously Fréchet differential functional with

$$
\left\langle\Phi^{\prime}(x), y\right\rangle=\int_{0}^{2 \pi}\left[x^{\prime}(t) y^{\prime}(t)+M x(t) y(t)-f\left(t, x^{+}(t)\right) y(t)\right] d t .
$$

Definition 2.1. A function $x$ is a classical solution to (2.1) if $x \in C^{2}([0,2 \pi]), x(0)=x(2 \pi)$, $x^{\prime}(0)=x^{\prime}(2 \pi)$, and

$$
x^{\prime \prime}(t)-M x(t)+f\left(t, x^{+}(t)\right)=0 \text { for every } t \in[0,2 \pi] .
$$

Definition 2.2. A function $x$ is a weak solution to (2.1) if $x \in X$ and

$$
\int_{0}^{2 \pi}\left[x^{\prime}(t) y^{\prime}(t)+M x(t) y(t)\right] d t=\int_{0}^{2 \pi} f\left(t, x^{+}(t)\right) y(t) d t
$$

for every $y \in X$.

Proposition 2.3. Classical and weak solutions to (2.1) coincide.

Lemma 2.4.([9]) Problem (2.1) is equivalent to the equation

$$
x(t)=T x(t)=\int_{0}^{2 \pi} G(t, s) f\left(s, x^{+}(s)\right) d s,
$$


where $m=\sqrt{(M)}$ and

$$
G(t, s)=G(|t-s|)= \begin{cases}\frac{e^{m(t-s)}+e^{m(2 \pi-t+s)}}{2 m\left(e^{2 m \pi}-1\right)}, & 0 \leq s \leq t \leq 2 \pi, \\ \frac{e^{m(s-t)}+e^{m(2 \pi-s+t)}}{2 m\left(e^{2 m \pi}-1\right)}, & 0 \leq t \leq s \leq 2 \pi .\end{cases}
$$

By computing, $G(t, s)$ has the following properties:

(a) $\frac{2 e^{m \pi}}{2 m\left(e^{2 m \pi}-1\right)}=G(\pi) \leq G(t, s) \leq G(0)=\frac{e^{2 m \pi}+1}{2 m\left(e^{2 m \pi}-1\right)}$;

(b) $\left|\frac{\partial}{\partial t} G(t, s)\right| \leq \frac{e^{2 m \pi}}{e^{2 m \pi}-1}$.

Proposition 2.5. A function $x$ is a classical solution if and only if $x$ is a fixed point of the operator $T$, that is $T x=x$, where $T$ is defined in Lemma 2.4 .

Lemma 2.6. For $x \in X$, then $\|x\|_{\infty} \leq \sqrt{2} \max \left\{(2 \pi M)^{-\frac{1}{2}},(2 \pi)^{\frac{1}{2}}\right\}\|x\|:=\Delta\|x\|$, where $\|x\|=$ $\max _{t \in[0,2 \pi]}|x(t)|$.

Proof. For $x \in X$, it follows from the mean value theorem that

$$
x(\tau)=\frac{1}{2 \pi} \int_{0}^{2 \pi} x(s) d s
$$

for some $\tau \in[0,2 \pi]$. Hence for $t \in[0,2 \pi]$, using Hölder inequality,

$$
\begin{aligned}
|x(t)| & =\left|x(\tau)+\int_{\tau}^{t} x^{\prime}(s) d s\right| \leq \frac{1}{2 \pi} \int_{0}^{2 \pi} x(s) d s+\int_{0}^{2 \pi}\left|x^{\prime}(s)\right| d s \\
& \leq(2 \pi)^{-\frac{1}{2}}\left(\int_{0}^{2 \pi}|x(s)|^{2} d s\right)^{\frac{1}{2}}+(2 \pi)^{\frac{1}{2}}\left(\int_{0}^{2 \pi}\left|x^{\prime}(s)\right|^{2} d s\right)^{\frac{1}{2}} \\
& \leq \sqrt{2} \max \left\{(2 \pi M)^{-\frac{1}{2}},(2 \pi)^{\frac{1}{2}}\right\}\|x\|,
\end{aligned}
$$

which completes the proof.

Lemma 2.7. (Th38.A [17]) For the functional $F: M \subseteq X \rightarrow[-\infty,+\infty]$ with $M \neq \varnothing, \min _{u \in M} F(u)=$ $\alpha$ has a solution in case the following hold:

(i) $X$ is a real reflexive Banach space;

(ii) $M$ is bounded and weak sequentially closed, i.e., by definition, for each sequence $\left(u_{n}\right)$ in $M$ such that $u_{n} \rightarrow u$ as $n \rightarrow \infty$, we always have $u \in M$;

(iii) $F$ is weak sequentially lower semi-continuous on $M$.

Lemma 2.8.([8]) Let $E$ be a Banach space and $\varphi \in C^{1}(E, R)$ satisfy $(P S)$ condition. Assume there exist $x_{0}, x_{1} \in E$, and a bounded open neighborhood $\Omega$ of $x_{0}$ such that $x_{1} \backslash \bar{\Omega}$ and

$$
\max \left\{\varphi\left(x_{0}\right), \varphi\left(x_{1}\right)\right\}<\inf _{x \in \partial \Omega} \varphi(x) .
$$


Let

$$
\Gamma=\left\{h \mid h:[0,1] \rightarrow E \text { is continuous and } h(0)=x_{0}, h(1)=x_{1}\right\}
$$

and

$$
c=\inf _{h \in \Gamma} \max _{s \in[0,1]} \varphi(h(s)) .
$$

The $c$ is a critical value of $\varphi$, that is, there exists $x^{*} \in E$ such that $\varphi^{\prime}\left(x^{*}\right)=\Theta$ and $\varphi\left(x^{*}\right)=c$, where $c>\max \left\{\varphi\left(x_{0}\right), \varphi\left(x_{1}\right)\right\}$.

\section{Main results}

Theorem 3.1. Suppose that $f(t, x)=a(t) x^{\mu-1}+g(t, x)$, where $\mu>2, a \in C([0,2 \pi],(0, \infty))$, $\min _{t \in[0,2 \pi]} a(t)>0$ and $g:[0,2 \pi] \times[0,+\infty) \rightarrow[0,+\infty)$ is continuous satisfying

$$
g(t, x) \leq b(t)+c(t) x
$$

$b, c \in C([0,2 \pi],[0,+\infty))$ satisfy $\|c\|_{\infty}<\min \left\{\frac{\mu-2}{2 \pi \mu} \Delta^{-2},\left(\Delta_{1} \Delta\right)^{-1}\right\}$ and

$$
\left(1-\Delta_{1} \Delta\|c\|_{\infty}\right)^{\frac{\mu-1}{\mu-2}}\left(\Delta_{1} \Delta^{\mu-1}\|a\|_{\infty}\right)^{-\frac{1}{\mu-2}}\left\{(\mu-1)^{-\frac{\mu-1}{\mu-2}}-(\mu-1)^{-\frac{1}{\mu-2}}\right\}+\Delta_{1}\|b\|_{\infty}<0,
$$

where $\Delta$ is defined in Lemma $2.6, \Delta_{1}=(2 \pi)^{\frac{3}{2}}\left[M(G(0))^{2}+\left(\frac{e^{2 m \pi}}{e^{2 m \pi}-1}\right)^{2}\right]^{\frac{1}{2}}$.

Then problem (1.1) has two positive solutions, at least one of which is in $B_{R}$, where $R=\left(\frac{1-\Delta_{1} \Delta\|c\|_{\infty}}{(\mu-1) \Delta_{1} \Delta^{\mu-1}\|a\|_{\infty}}\right)^{\frac{1}{\mu-2}}$.

Proof. We divide the proof into three steps.

Step 1 . We show that the functional $\Phi$ satisfies (PS) condition, that is, $\left(x_{n}\right)$ has a convergent subsequence whenever it satisfies $\Phi^{\prime}\left(x_{n}\right) \rightarrow 0$ as $n \rightarrow \infty$ and $\left\{\Phi\left(x_{n}\right)\right\}$ is bounded.

By (2.2), (2.3) and Lemma 2.1 we have

$$
\begin{aligned}
\left(\frac{\mu}{2}-1\right)\left\|x_{n}\right\|^{2}= & \mu \Phi\left(x_{n}\right)-\left\langle\Phi^{\prime}\left(x_{n}\right), x_{n}\right\rangle+\mu \int_{0}^{2 \pi} G\left(t, x_{n}^{+}(t) d t-\mu \int_{0}^{2 \pi}\left(g(t, 0), x_{n}^{-}(t)\right) d t\right. \\
& -\int_{0}^{2 \pi} g\left(t, x_{n}^{+}(t)\right) x_{n}(t) d t
\end{aligned}
$$

where $G(t, x)=\int_{0}^{x} g(t, s) d s$. Condition (3.1) means that

$$
G(t, x) \leq b(t) x+\frac{c(t)}{2} x^{2} .
$$

Substituting (3.1), (3.4) into (3.3), by Lemma 2.1, Lemma 2.6 we have

$$
\begin{aligned}
\left(\frac{\mu}{2}-1\right)\left\|x_{n}\right\|^{2} \leq & \mu \Phi\left(x_{n}\right)-\left\langle\Phi^{\prime}\left(x_{n}\right), x_{n}\right\rangle+2 \pi \mu\|b\|_{\infty}\left\|x_{n}^{+}\right\|_{\infty}+\frac{\mu}{2} \int_{0}^{2 \pi} c(t)\left(x_{n}^{+}(t)\right)^{2} d t \\
& +\int_{0}^{2 \pi} g\left(t, x_{n}^{+}\right) x_{n}^{-}(t) d t
\end{aligned}
$$




$$
\begin{aligned}
& \leq \mu \Phi\left(x_{n}\right)-\left\langle\Phi^{\prime}\left(x_{n}\right), x_{n}\right\rangle+2 \pi(\mu+1)\|b\|_{\infty}\left\|x_{n}\right\|_{\infty}+\pi \mu\|c\|_{\infty}\left\|x_{n}\right\|_{\infty}^{2} \\
& \leq \mu \Phi\left(x_{n}\right)-\left\langle\Phi^{\prime}\left(x_{n}\right), x_{n}\right\rangle+2 \pi(\mu+1) \Delta\|b\|_{\infty}\left\|x_{n}\right\|+\pi \mu \Delta^{2}\|c\|_{\infty}\left\|x_{n}\right\|^{2} .
\end{aligned}
$$

So

$$
\left(\frac{\mu}{2}-1-\pi \mu \Delta^{2}\|c\|_{\infty}\right)\left\|x_{n}\right\|^{2} \leq \mu \Phi\left(x_{n}\right)-\left\langle\Phi^{\prime}\left(x_{n}\right), x_{n}\right\rangle+2 \pi(\mu+1)\|b\|_{\infty} \Delta\left\|x_{n}\right\| .
$$

Since $\Phi\left(x_{n}\right)$ is bounded and $\Phi^{\prime}\left(x_{n}\right) \rightarrow 0$ as $n \rightarrow \infty$, we have that $\left(x_{n}\right)$ is bounded in $X$.

From the reflexivity of $X$, we may extract a weakly convergent sequence, that for simplicity, we call $\left(x_{n}\right), x_{n} \rightarrow x$. Following we will show that $\left(x_{n}\right)$ converges strongly to $x$. By (2.3) we have

$$
\left\|x_{n}-x\right\|^{2}=\left\langle\Phi^{\prime}\left(x_{n}\right), x_{n}-x\right\rangle-\left\langle\Phi^{\prime}(x), x_{n}-x\right\rangle+\int_{0}^{2 \pi}\left[f\left(t, x_{n}^{+}(t)\right)-f\left(t, x^{+}(t)\right)\right]\left(x_{n}(t)-x(t)\right) d t .
$$

Since $\Phi^{\prime}\left(x_{n}\right) \rightarrow 0$ by assumption, and $x_{n}-x$ is bounded in $X$, we deduce that $\left\langle\Phi^{\prime}\left(x_{n}\right), x_{n}-x\right\rangle \rightarrow$ 0 . By [12], $\left(x_{n}\right)$ converges uniformly to $x$ on $C([0,2 \pi])$. Noticing Lemma 2.1(iii), $\left(x_{n}^{+}\right)$converges uniformly to $x^{+}$on $C([0,2 \pi])$, which means

$$
\int_{0}^{2 \pi}\left[f\left(t, x_{n}^{+}(t)\right)-f\left(t, x^{+}(t)\right)\right]\left(x_{n}(t)-x(t)\right) d t \rightarrow 0 .
$$

Moreover, $x_{n} \rightarrow x$ and $\Phi^{\prime}(x) \in X^{*}$ means that $\left\langle\Phi^{\prime}(x), x_{n}-x\right\rangle \rightarrow 0$ as $n \rightarrow \infty$. Therefore, $\| x_{n}-$ $x \| \rightarrow 0$ as $n \rightarrow \infty$.

Step 2. There exists $R>0$ such that the functional $\Phi$ has a local minimum $x_{0} \in B_{R}:=\{x \in$ $X:\|x\|<R\}$.

Let $R>0$, which will be determined later. First we claim that the functional $\Phi$ has a minimum on $\bar{B}_{R}$. Clearly $\bar{B}_{R}$ is a bounded and weak sequentially closed. Following we will show that $\Phi$ is weak sequentially lower semi-continuous on $\bar{B}_{R}$. For this, let $\Phi^{1}(x)=\frac{1}{2} \int_{0}^{2 \pi}\left|x^{\prime}(t)\right|^{2}+$ $M|x(t)|^{2} d t=\frac{1}{2}\|x\|^{2}$ and $\Phi^{2}(x)=-\int_{0}^{2 \pi} F\left(t, x^{+}(t)\right)-f(t, 0) x^{-}(t) d t$, then $\Phi(x)=\Phi^{1}(x)+\Phi^{2}(x)$. By $x_{n} \rightarrow x$ on $X$ and Proposition 1.2 [12] we have $\left(x_{n}\right)$ uniformly converges to $x$ in $C([0,2 \pi])$. So $\Phi^{2}$ is weak sequentially continuous. Clearly $\Phi^{1}$ is continuous, which together with the convexity of $\Phi^{1}$ we have $\Phi^{1}$ is weak sequentially lower semi-continuous. Therefore, $\Phi$ is weak sequentially lower semi-continuous on $B_{R}$. Our claim follows from Lemma 2.7.

If $x_{0} \in \partial B_{R}$, then it is also a minimizer of $\left.\Phi\right|_{\partial B_{R}}$, so the gradient of $\Phi$ at $x_{0}$ point in the direction of the inward normal to $\partial B_{R}$, i.e.

$$
\Phi^{\prime}\left(x_{0}\right)=-\mu x_{0}
$$

for some $\mu \geq 0$, then $\left\langle\Phi^{\prime}\left(x_{0}\right), y\right\rangle=-\mu\left\langle x_{0}, y\right\rangle$ for all $y \in X$ holds. That is,

$$
x_{0}^{\prime \prime}(t)-M x_{0}(t)+\lambda f\left(t, x_{0}^{+}(t)\right)=0, \quad x_{0} \in X
$$

with $\lambda=\frac{1}{1+\mu} \in(0,1],\left\|x_{0}\right\|=R$ holds. 
Following we claim that for any $\lambda \in(0,1]$, the solution of (3.6) is not on $\partial B_{R}$, for which, we get a contradiction. In fact, by (3.1), Lemma 2.4, Lemma 2.6, it follows for $\|x\|=R$,

$$
\begin{aligned}
\|\lambda T x\| \leq & \left(\int_{0}^{2 \pi}\left[M|T x(t)|^{2}+\left|(T x)^{\prime}(t)\right|^{2}\right] d t\right)^{\frac{1}{2}} \\
= & \left(\int_{0}^{2 \pi} M\left|\int_{0}^{2 \pi} G(t, s) f\left(s, x^{+}(s)\right) d s\right|^{2}+\left|\int_{0}^{2 \pi}(\partial G / \partial t)(t, s) f\left(s, x^{+}(s)\right) d s\right|^{2} d t\right)^{\frac{1}{2}} \\
\leq & \left\{2 \pi\left[M\left(\max _{(t, s) \in[0,2 \pi]^{2}} G(t, s)\right)^{2}+\left(\max _{(t, s) \in[0,2 \pi]^{2}}(\partial G / \partial t)(t, s)\right)^{2}\right]\right\}^{\frac{1}{2}} \\
& \times\left|\int_{0}^{2 \pi} f\left(s, x^{+}(s)\right) d s\right| \\
< & (2 \pi)^{\frac{1}{2}}\left[M(G(0))^{2}+\left(\frac{e^{2 m \pi}}{e^{2 m \pi}-1}\right)^{2}\right]^{\frac{1}{2}}\left[2 \pi\|a\|_{\infty}\|x\|_{\infty}^{\mu-1}+2 \pi\|b\|_{\infty}+2 \pi\|c\|_{\infty}\|x\|_{\infty}\right] \\
\leq & \Delta_{1} \times\left[\Delta^{\mu-1}\|a\|_{\infty} R^{\mu-1}+\|b\|_{\infty}+\|c\|_{\infty} \Delta R\right] .
\end{aligned}
$$

Let

$$
\Lambda(R)=\Delta_{1} \times\left[\Delta^{\mu-1}\|a\|_{\infty} R^{\mu-1}+\|b\|_{\infty}+\|c\|_{\infty} \Delta R\right]-R .
$$

By computing we have $\Lambda^{\prime}(R) \leq 0$ for $R \in\left[0, R_{0}\right], \Lambda^{\prime}(R) \geq 0$ for $R \in\left[R_{0}, \infty\right)$, where $R_{0}=\left(\frac{1-\Delta_{1} \Delta\|c\|_{\infty}}{(\mu-1) \Delta_{1} \Delta^{\mu-1}\|a\|_{\infty}}\right)^{\frac{1}{\mu-2}}$. Moreover, by (3.2), we have $\Lambda\left(R_{0}\right)<0$. So let $R=R_{0},\|\lambda T x\|<\|x\|$ for $\|x\|=R$, that is, the solutions of $\lambda T x=x$ do not satisfh $\|x\|=R$. By Proposition 2.5, for amy $\lambda \in(0,1]$, (3.6) has no solution on $\partial B_{R}$. Therefore, $x_{0} \in B_{R}$ and hence it is a local minimizer of $\Phi$, and $\Phi\left(x_{0}\right)<\min _{x \in \partial B_{R}} \Phi(x)$.

Step 3. There exists $x_{1}$ with $\left\|x_{1}\right\|>R$ such that $\Phi\left(x_{1}\right)<\min _{x \in \partial B_{R}} \Phi(x)$.

Let $e(t)=\sin t \in X$, then

$$
\begin{aligned}
\Phi(\bar{\lambda} e) & =\frac{\bar{\lambda}^{2}}{2} \int_{0}^{2 \pi}\left[|\cos t|^{2}+M|\sin t|^{2}\right] d t-\int_{0}^{2 \pi}\left[F\left(t, \bar{\lambda}(\sin t)^{+}\right)-f(t, 0) \bar{\lambda}\left(\sin \left(\frac{2 \pi t}{T}\right)\right)^{-}\right] d t \\
& \leq \bar{\lambda}^{2}(1+M) \pi-\int_{0}^{2 \pi} \frac{\bar{\lambda}^{\mu} a(t)}{\mu}\left|(\sin t)^{+}\right|^{\mu} d t+\bar{\lambda} \int_{0}^{2 \pi} f(t, 0) d t .
\end{aligned}
$$

Since

$$
\int_{0}^{2 \pi}(\sin t)^{+} d t \leq(2 \pi)^{\frac{1}{\mu^{\prime}}}\left(\int_{0}^{2 \pi}\left|(\sin t)^{+}\right|^{\mu} d t\right)^{\frac{1}{\mu}}, \quad \text { where } \frac{1}{\mu}+\frac{1}{\mu^{\prime}}=1
$$

we have

$$
\Phi(\bar{\lambda} e) \leq \bar{\lambda}^{2}(1+M) \pi-\frac{\bar{\lambda}^{\mu}}{\mu} \min _{t \in[0,2 \pi]} a(t)(2 \pi)^{-\frac{\mu}{\mu^{\prime}}}\left(\int_{0}^{2 \pi}(\sin t)^{+} d t\right)^{\mu}+\bar{\lambda} \int_{0}^{T} f(t, 0) d t .
$$

Since $\mu>2$, we have $\lim _{\bar{\lambda} \rightarrow+\infty} \Phi(\bar{\lambda} e)=-\infty$. So there exists sufficiently large $\bar{\lambda}_{0}$ with $\left\|\bar{\lambda}_{0} e\right\|>R$ such that $\Phi\left(\bar{\lambda}_{0} e\right)<\min _{x \in \partial B_{R}} \Phi(x)$. 
Lemma 2.8 now gives the critical value

$$
c=\inf _{h \in \Gamma} \max _{t \in[0,1]} \Phi(h(t)),
$$

where

$$
\Gamma=\left\{h \mid h:[0,1] \rightarrow E \text { is continuous and } h(0)=x_{0}, h(1)=x_{1}\right\},
$$

that is, there exists $x^{*} \in X$ such that $\Phi^{\prime}\left(x^{*}\right)=0$. Therefore, $x_{0}, x^{*}$ are two critical points of $\Phi$, and hence they are classical solutions of (2.1) by Proposition 2.3. Lemma 2.2 means $x_{0}, x^{*}$ are two positive solutions of problem (1.1).

Remark 3.1. The assumptions on nonlinearity $f$ are different from those in [9]. There are functions satisfying Theorem 3.1 but not satisfying superlinear or sublinear in [9]. For example, let $M=4, \mu=3, f(t, x)=a(t) x^{2}+b(t)+c(t) x$, where $a(t)=\frac{1}{8 \pi^{\frac{5}{2}}}, b(t)=\frac{t}{720 \pi^{\frac{5}{2}}} c(t)=$ $\frac{1}{60 \pi^{3}}$ for $t \in[0,2 \pi]$. Then $f$ satisfies Theorem 3.1, but not satisfies theorem in [9]. Besides we get the existence of two positive solutions not one positive solution as in [9].

\section{References}

[1] Ravi P. Agarwal, Kanishka Perera and Donal O'Regan, Multiple positive solutions of singular and nonsingular discrete problems via variational methods, Nonlinear Anal. 58(2004), 69-73.

[2] F. Antonacci and P. Magrone, Second order nonautonomous systems with symmetric potential changing sign, Rend. Mat. Appl. 18(1998), 367-379.

[3] A. Cabada, The method of lower and upper solutions of second, third, fourth and higher order boundary value problems, J. Math. Appl. Anal. 185(1994), 302-320.

[4] G. L. Chen and Y. M. Long, Periodic solutions of second-order nonlinear Hamiltonian systems with superquadratic potentials having mean value zero, Chinese J. Contemp. Math. 19(1998), 333-342.

[5] James A. Clarkson, Uniformly convex spaces, Trans. Amer. Math. Soc. 40(1936), 396-414.

[6] Y. H. Ding, Existence and multiplicity results for homoclinic solutions to a class of Hamiltonian systems, Nonlinear Anal. 25(1995), 1095-1113.

[7] P. L. Felmer and E. A. de B. e Silva, Subharmonics near an equilibrium for some second-order Hamiltonian systems, Proc. Roy. Soc. Edinburgh Sect. A 123(1993), 819-834.

[8] Guo Dajun, Nonlinear Functional Analysis, Shandong science and technology Press, 1985.

[9] Daqing Jiang, On the existence of positive solutions to second order periodic BVPs, Acta Mathematica Scientia 18(1998), 31-35.

[10] Yuji Liu and Weigao Ge, On the positive periodic solutions of the nonlinear duffing equations with delay and variable coefficients, Bull. Belg. Math. Soc. 11 (2004), 441-455.

[11] Shiping Lu and Weigao Ge, Periodic solutions for a kind of Liénard equation with a deviating argument, J. Math. Anal. Appl. 289(2004), 231-243.

[12] J. Mawhin and M. Willem, Critical Point Theory and Hamiltonian Systems, Springer-Verlag, Berlin, 1989.

[13] J. J. Nieto, Nonlinear second order periodic boundary value problems with Carathédory functions, Appl. Anal. 34(1989), 111-128.

[14] P. Rabinowitz, Periodic solutions of Hamiltonian systems, Comm. Pure Appl. Math. 31(1978), 157184. 
[15] P. H. Rabinowitz, Minimax Methods in Critical Point Theory with Applications to Differential Equations, in: CBMS Regional Conf. Ser. in Math. 65, American Mathematical Society, Providence, RI, 1986.

[16] Chun-Lei Tang and Xing-Ping Wu, Periodic solutions for second order Hamiltonian systems with a change sign potentical, J. Math. Anal. Appl. 292(2004), 506-516.

[17] E. Zeidler, Nonlinear Functional Analysis and its Application, Vol. III, Springer, 1985.

School of Science, Beijing University of Posts and Telecommunications, Beijing 100876, P.R. China.

Department of Applied Mathematics, Beijing Institute of Technology, Beijing 100081, P.R. China. 